\title{
The leading-edge vortex and quasisteady vortex shedding on an accelerating plate
}

\author{
Kevin K. Chen, ${ }^{1, a)}$ Tim Colonius, ${ }^{1, b)}$ and Kunihiko Taira ${ }^{2}$ \\ ${ }^{1}$ Department of Mechanical Engineering, California Institute of Technology, \\ Pasadena, California 91125, USA \\ ${ }^{2}$ Department of Mechanical and Aerospace Engineering, Princeton University, \\ Princeton, New Jersey 08544, USA
}

(Received 3 August 2009; accepted 5 January 2010; published online 10 March 2010)

\begin{abstract}
A computational inquiry focuses on leading-edge vortex (LEV) growth and shedding during acceleration of a two-dimensional flat plate at a fixed $10^{\circ}-60^{\circ}$ angle of attack and low Reynolds number. The plate accelerates from rest with a velocity given by a power of time ranging from 0 to 5. During the initial LEV growth, subtraction of the added mass lift from the computed lift reveals an LEV-induced lift augmentation evident across all powers and angles of attack. For the range of Reynolds numbers considered, a universal time scale exists for the peak when $\alpha \geq 30^{\circ}$, with augmentation lasting about four to five chord lengths of translation. This time scale matches well with the half-stroke of a flying insect. An oscillating pattern of leading- and trailing-edge vortex shedding follows the shedding of the initial LEV. The nondimensional frequency of shedding and lift coefficient minima and maxima closely match their values in the absence of acceleration. These observations support a quasisteady theory of vortex shedding, where dynamics are determined primarily by velocity and not acceleration. Finally, the nondimensional vortex formation time is found to be a function of the Reynolds number, but only weakly when the Reynolds number is high. (C) 2010 American Institute of Physics. [doi:10.1063/1.3327282]
\end{abstract}

\section{INTRODUCTION}

Unsteady effects pervade open problems in flappingwing flight. These effects, including vortex growth and shedding, are largely absent in classical fixed-wing theory. Their quantification is crucial for understanding flapping-wing flight mechanisms.

In particular, leading-edge vortices (LEVs)_low pressure regions of circulating fluid above a wing at an angle of attack-play a crucial role in the lift of flapping-wing birds and insects. ${ }^{1-4}$ Typical flapping-wing animals fly in a Reynolds number range around 10-10000 and employ high angles of attack. ${ }^{1}$ Their wings' sharp edges and high angles of attack create LEVs that enhance the lift force exerted on the wing.

Dickinson and Götz ${ }^{5}$ performed an experimental study on a nominally two-dimensional (2D) airfoil, employing impulsively started translations over a wide range of angles of attack. This study revealed a large peak in the lift coefficient after about half a chord length of displacement, corresponding to the growth and attachment of the startup LEV. Comparisons between lift forces at two chord lengths of displacement and seven chord lengths revealed that the former typically experienced up to $80 \%$ more lift. The fact that flapping wings tend to displace only two to four chord lengths during each half-stroke implies that flapping-wing animals

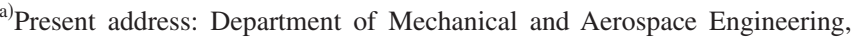
Princeton University.

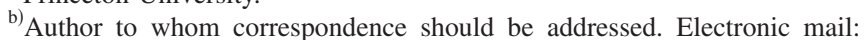
colonius@caltech.edu.
}

can benefit from the large lift augmentation of initial LEV growth.

Taira and Colonius ${ }^{4}$ computed impulsively started threedimensional (3D) flows at $\mathrm{Re}=300$ and 500. In this study, the aspect ratio, angle of attack, and geometry of a flat plate were varied. It was found that the aspect ratio had a large effect on the qualitative nature of the wake, as well as on the forces the plate applied to the fluid. In addition, a large peak in lift coefficient was detected at a displacement of 1.7 chord lengths, regardless of aspect ratio, angle of attack, and geometry.

A number of studies have focused on the attachment and prolongation of the initial LEV because of the resulting favorable lift augmentation results. Existing literature, including Birch et al. ${ }^{1}$ and Taira and Colonius, ${ }^{4}$ credit the attachment of 3D startup LEVs primarily to spanwise flow. Such flow toward the wingtip tends to move momentum and vorticity out of the LEV, giving it stability. Typically, the spanwise flow stretches the LEV from the wing root to a point about three quarters to the wingtip, where the vortex line moves off the wing and into the wake. ${ }^{2}$ Interestingly, Birch et al. ${ }^{1}$ found that no spanwise flow exists in the LEV core at $\mathrm{Re} \approx 100$ and that the mode of vorticity transport out of the LEV depends on the Reynolds number.

Pullin and Wang ${ }^{6}$ (hereafter referred to as PW) numerically computed 2D solutions for a flat plate accelerating from rest at moderate to high angles of attack and low Reynolds numbers. A power law of the form $\hat{U}(\hat{t})=B \hat{t}^{m}$ was used for the plate velocity, and the study focused on the transient response of the airfoil $(\hat{t}<2$ in this case, with $\hat{U}$ and $\hat{t}$ non- 
dimensionalized by some reference velocity and the chord length). The early transient results of PW matched somewhat closely with Wagner's theory of unsteady airfoils. ${ }^{7}$ However, the peak in lift coefficient found by Taira and Colonius, ${ }^{4}$ Dickinson and Götz, ${ }^{5}$ and others was not emphasized in the results of PW when a power of $m=1$ was applied.

Despite the work of PW, the existing literature has not systematically examined the effects of airfoil acceleration on dynamic forces and vortical structures. For instance, PW developed a theory that can be applied to any power law velocity, but only focused numerical tests on linear velocities (i.e., $\hat{U}=B \hat{t}$ ). In addition, the theory of PW is valid only in the early transient response of a flat plate to motion. Therefore, this computational study is undertaken to provide a careful and broad characterization of acceleration effects through the use of power law velocity relations. Special focus is placed on the kinematics and dynamics of the initial LEV growth. We expand on the research of PW by simulating a wide range of velocity powers, and by analyzing lift force responses up to large times. Furthermore, we highlight the lift coefficient peak in this study. Although the wing studied here is considered fixed instead of flapping, its setup yields important dynamics relevant to flapping-wing flight.

In our study, a $2 \mathrm{D}$ plate is displaced with power law velocities of the form $U(t) / U_{\text {ref }}=\left(t U_{\text {ref }} / c\right)^{\beta}$ or of the dimensionless form

$$
\hat{U}(\hat{t})=\hat{t}^{\beta} .
$$

Here, $\hat{U} \equiv U / U_{\text {ref }}$ and $\hat{t} \equiv t U_{\text {ref }} / c \geq 0$ for some reference velocity $U_{\text {ref }}$ and chord $c$. The power law form is chosen because of the versatility with which it can be applied and because different accelerations can be investigated by simply changing $\beta$. The power $\beta$ ranges from 0 to 5 . Special focus is placed on an angle of attack of $\alpha=30^{\circ}$ and a reference Reynolds number $\operatorname{Re}_{\text {ref }} \equiv U_{\text {ref }} c / \nu=100$. Simulations are run long enough to consider both transient and long-time responses.

Since the simulations are 2D, we ignore tip effects, spanwise transport of vorticity, and other 3D phenomena. Threedimensionality cannot be ignored at sufficiently high Re, but we do not address these issues in this paper.

\section{SETUP}

\section{A. Numerical method}

The incompressible Navier-Stokes equations are solved using an immersed boundary (IB) projection method. ${ }^{8,9}$ The solid body is represented on an otherwise regular Cartesian mesh by a set of discrete forces that are in turn regularized (smeared) on the grid. At the discrete body points, the no-slip condition is exactly enforced. For the present simulations, the acceleration of the plate is handled by a change of reference frame to one moving with the plate, such that an accelerating freestream flow is imposed. An additional fictitious body force is added to the momentum equation to account for the noninertial reference frame. The equations are discretized with a second-order finite-volume method and a stream function-vorticity approach is used on a staggered grid arrangement. The divergence free constraint is exactly satisfied (to machine precision). Further details regarding the numerical method can be found in the aforementioned references.

In these simulations, an infinitely thin flat plate is used for the geometry. At the low Reynolds numbers and high angles of attack considered here, the airfoil cross section is relatively unimportant to the separated flow dynamics. The plate is regularized by the IB method such that its thickness scales with the computational grid spacing. Comparisons with low Reynolds number towing tank experiments (for 3D foils) confirms the efficacy of this approach. ${ }^{4}$

The computational domain extends overall to a distance of 64 and 32 chord lengths in the streamwise and normal directions. Our IB method uses a series of overlapping, consecutively larger and coarser domains. The smallest domain with finest resolution extends to four and two chord lengths in the streamwise and normal directions. The grid spacing $h / c$ on this finest grid is 0.00625 or 0.01 for the majority of cases studied. Selected cases are run on a coarser grid with $h / c=0.0125$ and 0.02 to test the grid convergence. With one caveat, the results presented in the paper are converged. The caveat is that for the fastest accelerations and higher value of $\mathrm{Re}_{\text {ref }}$ of 200 , the solution is only marginally resolved at late times. This does not have any significant impact on the overall conclusions of the study.

An additional validation is performed by comparing with theoretical results for the early time accelerations and is given in Sec. II C.

\section{B. Nondimensionalization}

We wish to apply a plate velocity of the dimensional power law form $U(t)=k t^{\beta}$. The $t^{\beta}$ form provides the power law and $k$ is a multiplicative factor that we need to generalize this form to all power law velocities. Let $c$ be the chord length and $U_{\text {ref }}$ be the velocity scale and let $k \equiv U_{\text {ref }}^{1+\beta} / c^{\beta}$. Then

$$
\begin{aligned}
& U(t)=\frac{U_{\mathrm{ref}}^{1+\beta}}{c^{\beta}} t^{\beta}, \\
& \frac{U}{U_{\mathrm{ref}}}=\left(\frac{t U_{\mathrm{ref}}}{c}\right)^{\beta},
\end{aligned}
$$

or alternatively,

$$
\hat{U}=\hat{t}^{\beta} .
$$

Keeping in mind that $U=U\left(c, t, U_{\text {ref }}, \beta\right)$, we can fully resolve the force per span $F_{L}$ by

$$
F_{L}=F_{L}\left(t ; \rho, \mu, c, U_{\text {ref }}, \alpha, \beta\right) .
$$

In nondimensional form, we find

$$
C_{L}=C_{L}\left(\hat{t} ; \operatorname{Re}_{\mathrm{ref}}, \alpha, \beta\right) .
$$

In this study, we apply $\alpha=10^{\circ}$ to $60^{\circ}$ and use $\operatorname{Re}_{\text {ref }}=10,100$, and 200; we report mostly on the results from $\alpha=30^{\circ}$ and $\operatorname{Re}_{\text {ref }}=100$. 
TABLE I. Table of nondimensional groups.

\begin{tabular}{lcc}
\hline \hline Parameter & General definition & Specific form \\
\hline$\alpha$ & Angle of attack & $10^{\circ}-60^{\circ}$ \\
$\beta$ & Power & $0-5$ \\
$\hat{t}$ & $t U_{\text {ref }} / c$ & $t U_{\text {ref }} / c$ \\
$\tilde{t}$ & $(1 / c) \int_{0}^{t} U(\tau) d \tau$ & $\hat{t}^{\beta+1} /(\beta+1)$ \\
$\operatorname{Re}_{\mathrm{ref}}$ & $\rho U_{\mathrm{ref}} c / \mu$ & $\operatorname{Re}$ at $\hat{t}=1$, any $\beta$ \\
$\hat{U}$ & $U(t) / U_{\mathrm{ref}}$ & $\operatorname{Re}(t) / \operatorname{Re}$ ref or $\hat{t}^{\beta}$ \\
$C_{D}$ & $F_{D} /\left[\frac{1}{2} \rho U^{2}(t) c\right]$ & $2 F_{D} c^{2 \beta-1} /\left(\rho U_{\mathrm{ref}}^{2 \beta+2} t^{2 \beta}\right)$ \\
$C_{L}$ & $F_{L} /\left[\frac{1}{2} \rho U^{2}(t) c\right]$ & $2 F_{L} c^{2 \beta-1} /\left(\rho U_{\mathrm{ref}}^{2 \beta+2} t^{2 \beta}\right)$ \\
\hline \hline
\end{tabular}

Although the computation uses a nondimensional time $\hat{t} \equiv t U_{\text {ref }} / c$, this form is based on the reference velocity, so physical time scales in $\hat{t}$ are sensitive to the instantaneous velocity of the airfoil. To nondimensionalize time in a way that is (as we show) generally insensitive to instantaneous velocity, it is useful to use units of chord lengths. This parameter is given by

$$
\tilde{t} \equiv \frac{1}{c} \int_{0}^{t} U(\tau) d \tau .
$$

Using the dimensional speed power law given by Eq. (2), the integral evaluates to $\tilde{t}=\hat{t}^{\beta+1} /(\beta+1)$.

All nondimensional parameters are summarized in Table I.

\section{Validation}

An additional check of the numerics and their implementation is made by comparing preliminary results to Wagner's inviscid theory of unsteady airfoils. ${ }^{7}$ Wagner's theory itself was verified in his own experiments, and also in a 2D com- putational study by $\mathrm{PW},{ }^{6}$ which showed good agreement in accelerating flows up to $\operatorname{Re}_{\text {ref }}=800$ and $\hat{t}=1.8$ at $\alpha=30^{\circ}$. For power law velocities of the form $\hat{U}=\hat{t}^{\beta}$ and an angle of attack of $30^{\circ}$, Wagner's theory gives the lift coefficient as

$$
C_{L}=\frac{\pi}{2}+\frac{\sqrt{3} \pi \beta}{8(\beta+1) \tilde{t}} .
$$

Computational simulations are run at $\hat{U}(\hat{t})=\hat{t}^{\beta}$, $\operatorname{Re}_{\text {ref }}=100$, and $\alpha=30^{\circ}$ for $\beta=0.5$ and $\beta=2$. Tests are also conducted at $\beta=0.5$ for $\operatorname{Re}_{\text {ref }}=10$ and 200. The results are shown in Fig. 1. In general, the IB method provides very good agreement with Wagner's theory during the early transient stage that the theory was developed to model. In the case when $\operatorname{Re}_{\text {ref }}=10$, the agreement is not as strong, but this is due to viscous effects that Wagner's inviscid theory does not capture. At $\mathrm{Re}_{\mathrm{ref}}=200$, the upward deviation from the theory is the result of a more pronounced lift augmentation compared with $\mathrm{Re}_{\mathrm{ref}}=10$ and 100 . Overall, the good match at low $\hat{t}$ provides confidence that the IB algorithm is properly implemented for accelerating bodies.

\section{RESULTS}

We begin by surveying the results for $\mathrm{Re}_{\mathrm{ref}}=100$ with $\alpha=30^{\circ}$. Figure 2 shows the transient response of the accelerating flat plate. In Fig. 2(a), four of the velocities applied are shown, ranging from an impulsive start at $\beta=0.0$ to a relatively strong power law at $\beta=1.5$. Computations are run at powers up to $\beta=5.0$. Low values of $\beta$ exhibit strong transient acceleration and weaker acceleration later on; this is similar to the impulsively started motion used in many prior studies. ${ }^{4,5}$ Higher values of $\beta$ exhibit weak transient accel-
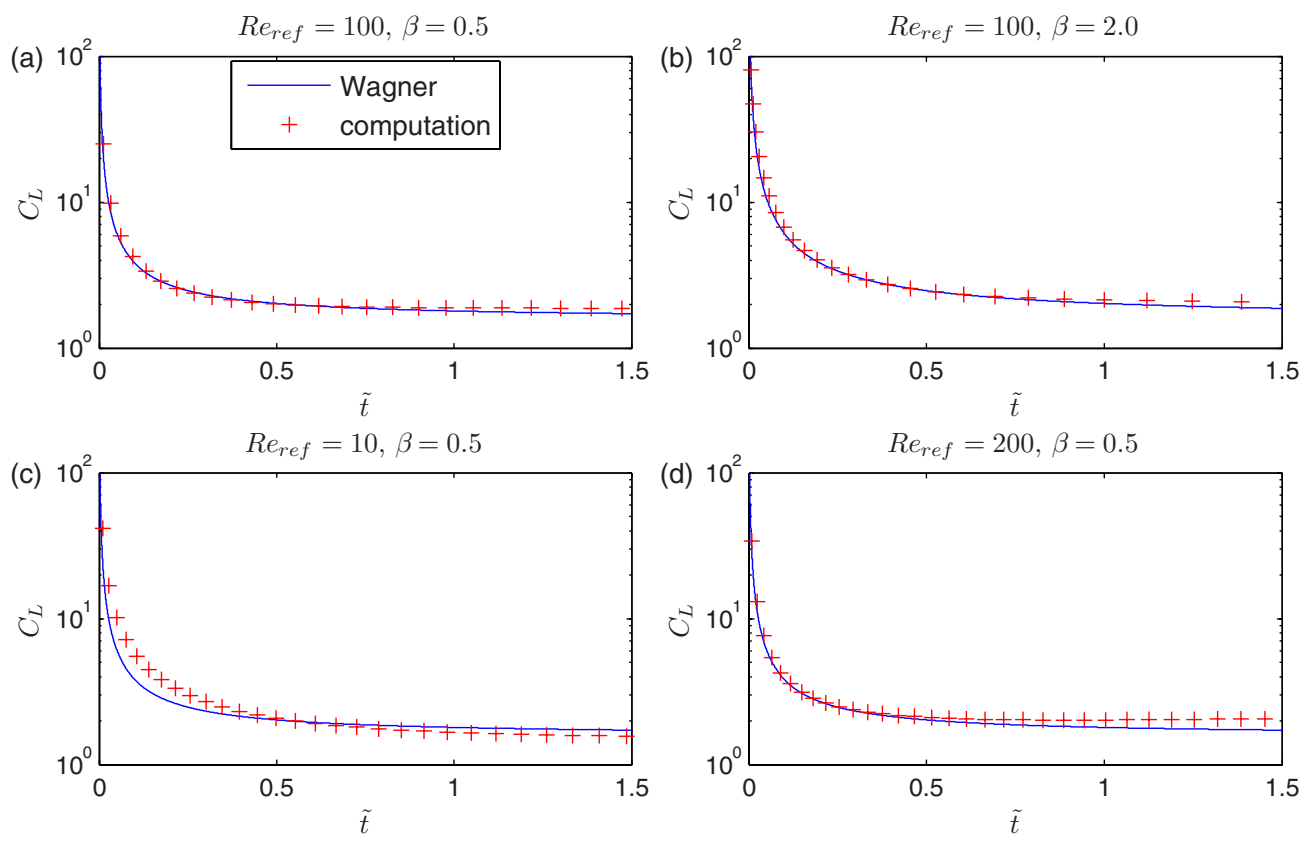

FIG. 1. (Color online) Comparison to Wagner's theory, with $\hat{U}(\hat{t})=\hat{t}^{\beta}$ and $\alpha=30^{\circ}$. Plots (a) and (b) are at a reference Reynolds number of 100 . (c) is at a lower $\mathrm{Re}_{\mathrm{ref}}=10$ and (d) is at a higher $\mathrm{Re}_{\mathrm{ref}}=200$. 
(a)
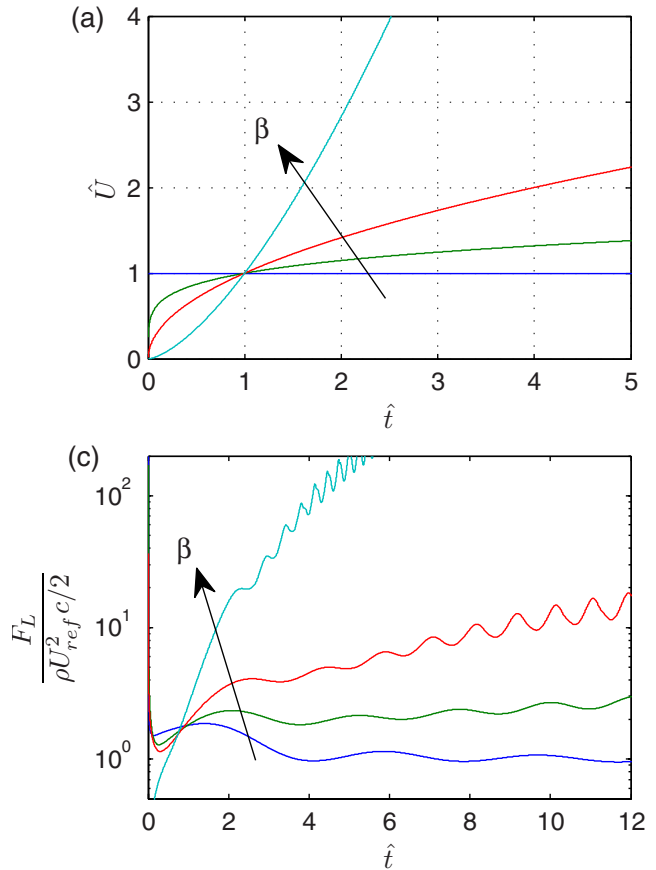

(b)
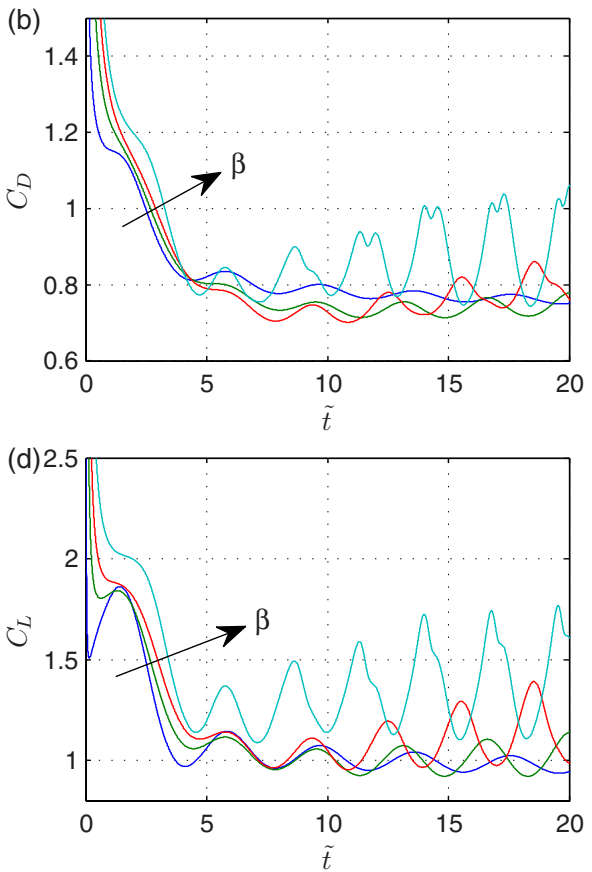

FIG. 2. (Color online) The early response of the accelerating airfoils at $\operatorname{Re}_{\mathrm{ref}}=100$ and $\alpha=30^{\circ}$. Shown are $\beta=0.0,0.2,0.5$, and 1.5 . (a) Some velocity power laws applied to the airfoil. Lower values of $\beta$ resemble Heaviside step motion, i.e., $\beta=0.0$. (b) Drag force and time normalized by $\hat{U}(\hat{t})$. (c) Lift force and time normalized by $U_{\text {ref. }}$ (d) Lift force and time normalized by $\hat{U}(\hat{t})$. By using this normalization, the multiplicative dependence of lift, drag, and time scales on $\hat{U}$ is included. The remaining difference between the curves is the result of additional factors such as acceleration and instantaneous Reynolds number.

eration and stronger acceleration soon afterwards. Figure 2(b) shows the evolution of the drag coefficient $C_{D}$ with time, and the effect of a change in $\beta$.

In Fig. 2(c), the time evolution of a constant-scaled lift force, $F_{L} /\left(\rho U_{\text {ref }}^{2} c / 2\right)$, is depicted as a function of a constantscaled time $\hat{t}$. However, this form is not necessarily useful, because dimensional analysis predicts that lift should scale by $\hat{U}^{2}(\hat{t})$ and time by $\hat{U}^{-1}(\hat{t})$, both of which continually change over time. Therefore, we consider the lift coefficient $C_{L}$ defined in Table I and nondimensional chord lengths of displacement $\tilde{t}$; this is shown in Fig. 2(d). The fact that the transient responses in Fig. 2(d) do not converge indicates that acceleration cannot be ignored. This has been discussed in recent studies that reject the so-called "quasisteady state model" of unsteady aerodynamics. ${ }^{2,5}$ All $C_{L}$ curves begin with an early transient response $C_{L} \sim \widetilde{t}^{-1}$ consistent with the airfoil theories of Wagner and $\mathrm{PW} ;^{6}$ this term arises from added mass considerations. During the early growth of the initial separation bubble, the lift coefficient continues to drop roughly as $\widetilde{t}^{-1}$, but a positive upturn is easily visible in the $C_{L}$ curves. The maximum lift augmentation (which will be more rigorously defined in Sec. III B) occurs at an early time, ranging from 1.4 chord lengths at $\beta=0$ to 2.5 chord lengths at $\beta=5$. After this point, an oscillating pattern of leadingand trailing-edge vortices is established; the oscillation maximum increases quickly at first (e.g., $8<\tilde{t}<30$ at $\beta=0.5$ ) before reaching a quasisteady state. During these fluctuations, the oscillation minimum remains largely unchanged.

\section{A. Early transients}

Wagner $^{7}$ and PW (Ref. 6) provide two models for the early force response on the accelerating flat plate. The former only considers trailing-edge vortex shedding, neglects vortex sheet roll-up, and assumes that vorticity advects at the stream speed. The theory of PW models separated flow at the leading edge using spiral vortex sheets. Both theories assume inviscid conditions, ${ }^{6}$ which make them applicable only during the early transient response. Once the size of the LEV becomes non-negligible, the inviscid assumption no longer holds, and both theories fail.

Wagner's theory gives the lift forces on a 2D airfoil at small $t$ and $\alpha$ as

$$
F_{y^{\prime}}=\frac{1}{2} \pi \rho c U^{2}(t) \sin \alpha+\frac{d}{d t}\left[m_{21}^{\prime} U(t)\right]
$$

where $x^{\prime}$ is opposite to the direction of the moving body, $x$ is aligned with the airfoil chord, and $\alpha$ is the clockwise angle of $x$ from $x^{\prime}{ }^{6}$ Assuming an elliptical airfoil with semimajor axis $a$ in the $x$-direction and semiminor axis $b$ in the $y$-direction, the added mass tensor in the $x^{\prime} y^{\prime}$-frame is $\mathbf{m}^{\prime}$ $=\mathbf{R} \mathbf{m} \mathbf{R}^{-1}$; the added mass tensor $\mathbf{m}$ in the $x y$-frame and the rotation matrix $\mathbf{R}$ are given by

$$
\mathbf{m}=\left[\begin{array}{cc}
-\pi \rho b^{2} & 0 \\
0 & -\pi \rho a^{2}
\end{array}\right], \quad \mathbf{R}=\left[\begin{array}{cc}
\cos \alpha & -\sin \alpha \\
\sin \alpha & \cos \alpha
\end{array}\right] .
$$

With $U(t) / U_{\text {ref }}=\left(t U_{\text {ref }} / c\right)^{\beta}, a=c / 2, b=0$, and $\alpha=30^{\circ}$, the lift coefficient is 

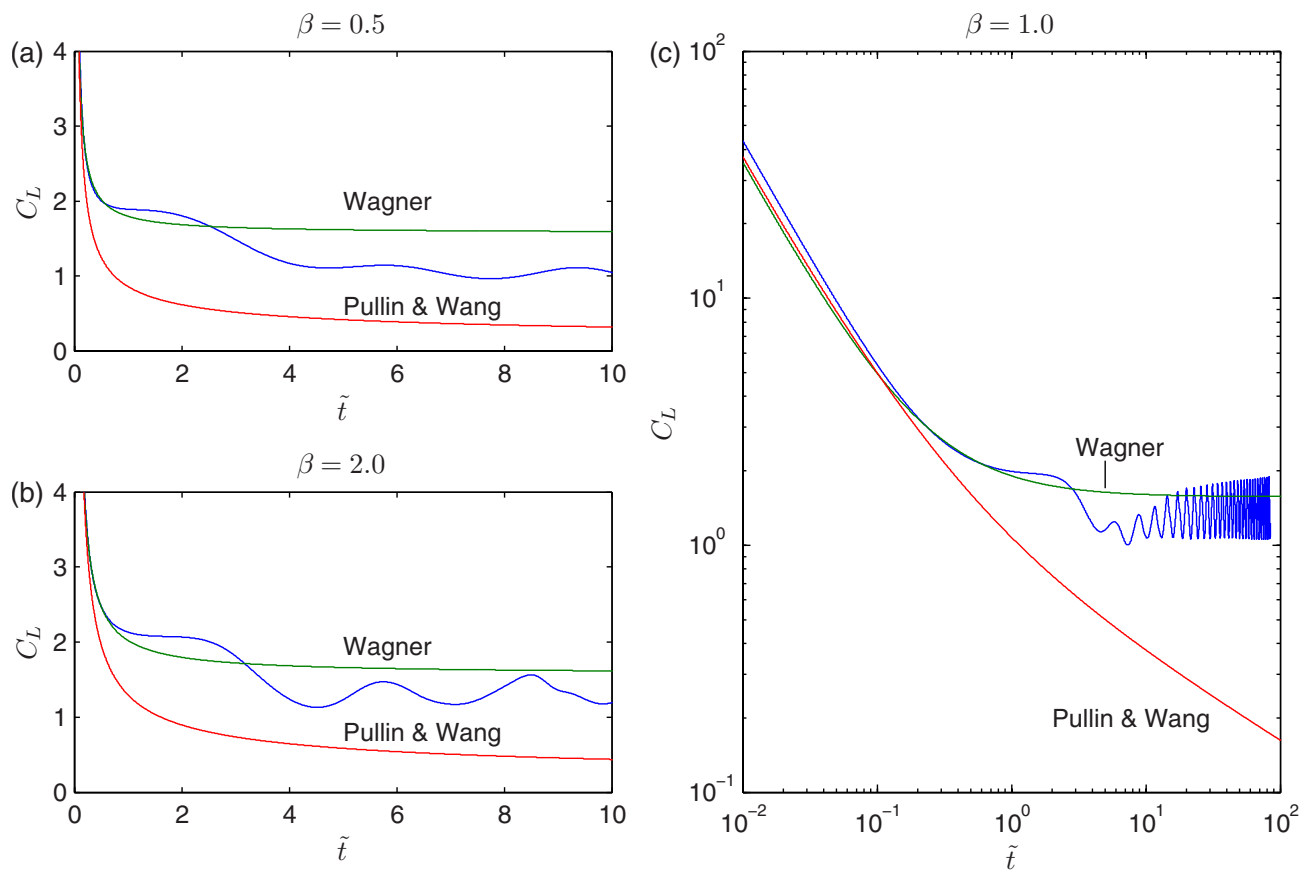

FIG. 3. (Color online) Comparison of computational results, the theory of Wagner, and the theory of $\mathrm{PW}$ at $\mathrm{Re}_{\mathrm{ref}}=100$ and $\alpha=30^{\circ}$. (a) $\beta=0.5$, (b) $\beta=2.0$, and (c) $\beta=1.0$, as a $\log -\log$ plot. This view emphasizes the relation between $C_{L}$ and $\tilde{t}$ at very early times.

$$
C_{L}=\frac{F_{y^{\prime}}}{\rho U^{2}(t) c / 2}=\frac{\pi}{2}+\frac{\sqrt{3} \pi \beta}{8(\beta+1) \tilde{t}} .
$$

Using our current notation, the theory of PW gives the lift coefficient in this same scenario as

$$
\begin{aligned}
C_{L}= & \frac{4(5 \beta+2)}{3}\left(\frac{3}{4(\beta+1)}\right)^{2 / 3} J_{0} \\
& \times \sin ^{5 / 3} \alpha \cos \alpha \Re\left[\int_{0}^{1} \omega_{0}^{1 / 2}(\lambda) d \lambda\right] \hat{t}^{-(\beta+1) / 3} \\
& +\frac{\pi \beta}{2} \hat{t}^{-\beta-1} \cos \alpha \sin \alpha .
\end{aligned}
$$

The parameter $J_{0}$ and the real part of the complex shape function integral are approximated as 2.2 and 0.32 , respectively. ${ }^{6}$ Hence, at $\alpha=30^{\circ}$,

$$
C_{L}=\frac{0.2114(5 \beta+2)}{(\beta+1) \tilde{t}^{1 / 3}}+\frac{\sqrt{3} \pi \beta}{8(\beta+1) \tilde{t}} \text {. }
$$

The $\widetilde{t}^{-1}$ added mass term is identical in the two theories, but Wagner's theory has an additional constant term instead of a $\tilde{t}^{-1 / 3}$ term.

The computational results for three different values of $\beta$ are compared with the theories of Wagner and PW. Figures 3(a) and 3(b) confirm that the computed results depart quickly from inviscid theory. This is expected, as the inviscid assumption applies only during the very early times when vorticity generated at the plate's surface has not yet diffused into the flow. The log-log axes in Fig. 3(c) emphasize the fact that the $\tilde{t}^{-1}$ term is the driving term at low $\tilde{t}$ in both theories. The comparison between computational results, the theory of Wagner, and the theory of PW are shown for a translating ellipse in the paper of PW (Ref. 6) with unscaled axes.

\section{B. Transients}

We now shift to the later development of the initial LEV. The lift augmentation is characterized here by subtracting the added mass lift from the computed lift, that is,

$$
C_{L}^{\prime}=C_{L}-\frac{\pi \sin (2 \alpha) \beta}{4(\beta+1) \tilde{t}} .
$$

We define $C_{L}^{\prime}$ this way because the lift response at early times is dominated by added mass effects, as described in Sec. III A and in Fig. 3(c). Without the added mass term, $C_{L}^{\prime}$ reflects the effect of the LEV, trailing edge vortex, and potential flow on the lift. The potential flow only adds a timeindependent constant to the lift coefficient.

The augmented lift $C_{L}^{\prime}$ is shown in Fig. 4(a) for $\operatorname{Re}_{\text {ref }}=100, \alpha=30^{\circ}$, and $\tilde{t}<5$. By subtracting the added mass lift from the true lift, the peaks in Fig. 2(d) become clear for all values of $\beta$; this can be thought of as a plot of the LEV contribution to the lift. The peak exists for all $\beta$, and it can be deduced that at this angle of attack, the lift augmentation universally begins near a point when the lift coefficient is close to its Wagner theoretical value of $\pi / 2$. Nevertheless, it is also evident that the exact nature of the peak depends on $\beta$.

Figures 4(b)-4(d) depict the dependence of the peak characteristics on $\beta$. The first shows the value of $C_{L}^{\prime}$ at the minima and the maximum. The magnitude of the maximum lift augmentation dips when $\beta$ is near 0.6. Figure 4(c) indicates that the maximum only occurs in a small time interval, 

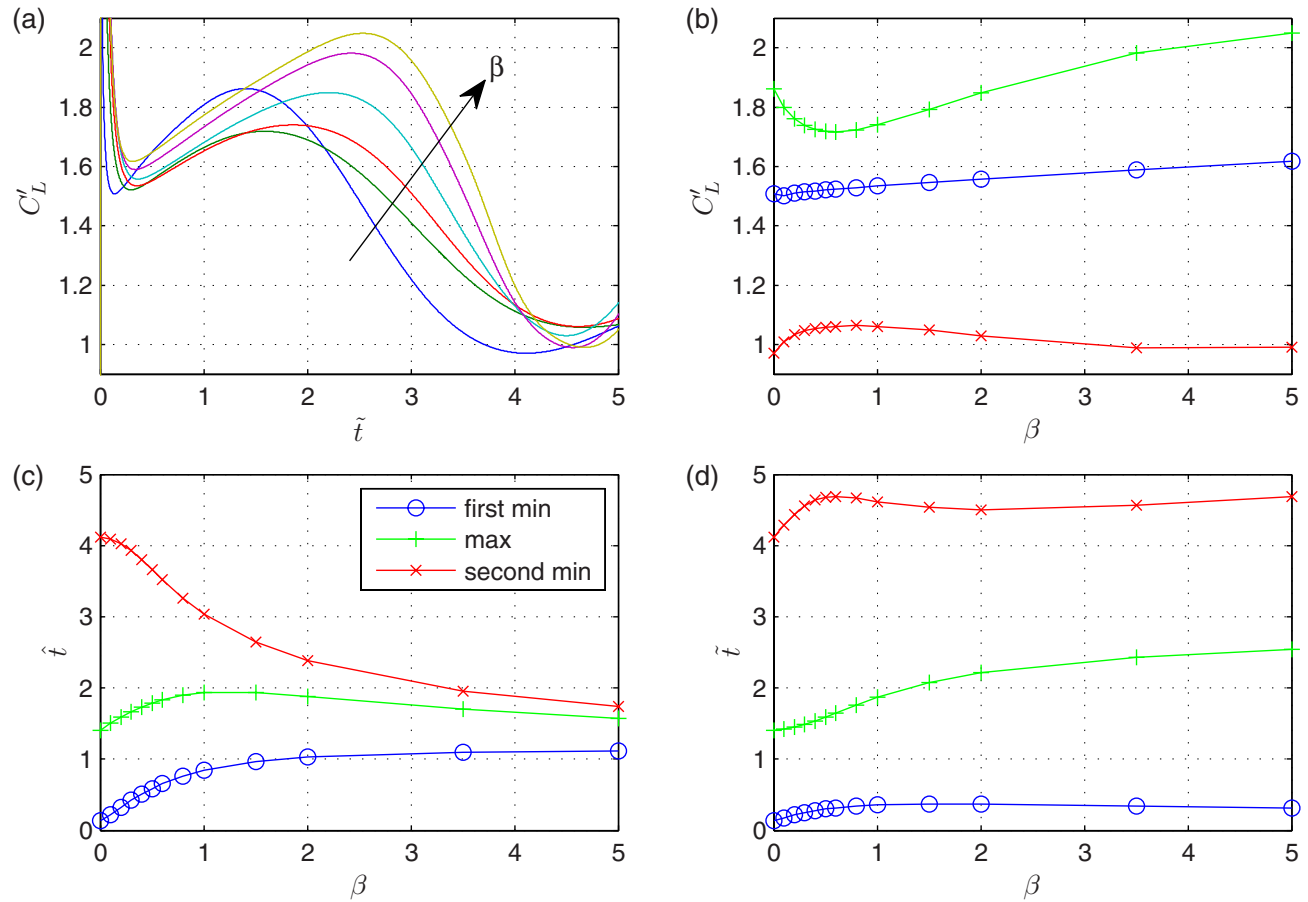

FIG. 4. (Color online) Analysis of the lift augmentation peak during the growth of the first LEV at $\operatorname{Re}_{\text {ref }}=100$ and $\alpha=30^{\circ}$. (a) The viscous contribution to the lift coefficient found by subtracting the added mass lift from computational results. Plots are shown for $\beta=0.0,0.5,1.0,2.0,3.5$, and 5.0. (b) The value of $C_{L}^{\prime}$ at which $C_{L}^{\prime}$ reaches its first minimum, its maximum, and its second minimum. (c) The value of $\hat{t}$ at the same points. (d) The value of $\tilde{t}$ at the same points.

from $\hat{t}=1.41$ at $\beta=0$ to $\hat{t}=1.93$ at $\beta=1.5$. However, it may be more meaningful to view the relation in terms of chord lengths $\tilde{t}$, instead of laboratory time $\hat{t}$. In terms of chord lengths, Fig. 4(d) implies that the time scale at which the peak begins and ends is consistent across different values of $\beta$. Although the maximum of the lift augmentation is observed to occur later with increased $\beta$, the temporal bounds of the $C_{L}^{\prime}$ peak occurs at "universal" times, completing around $\tilde{t}=4.5$.

It is interesting to note that the lift augmentation curves nearly collapse when only $0.0 \leq \beta \leq 1.0$ is considered, that is, when we only consider accelerations between impulsive and linear acceleration. Figure 5(a) is a reproduction of Fig. 4(a) that focuses on this low $\beta$ range at $\mathrm{Re}_{\mathrm{ref}}=100$. At $\mathrm{Re}_{\mathrm{ref}}$ $=200$, as in Fig. 5(b), the augmentation is more pronounced, but the curves collapse equally well. In addition, the peak is observed to begin near $\tilde{t} \approx 0.25$, and again, end in the vicinity of $\tilde{t}=4.0-4.5$. This supports the notion of a universal displacement scale for the lift augmentation observed during initial LEV growth. This $\tilde{t}$ scale appears to apply across $\operatorname{Re}_{\text {ref }}$ and $\beta$.

Some additional insight may be gained by varying the angle of attack from $\alpha=10^{\circ}$ to $\alpha=60^{\circ}$. For instance, $\mathrm{PW}^{6}$ predicted that the vortex lift should be maximized at $\alpha$ $=52.2^{\circ}$. The lift augmentation for this range of $\alpha$ is shown in Fig. 6 for $\beta$ ranging from 0.5 to 2.0. A few features are universally present across this range of $\beta$. First, there are no clearly observable lift augmentation peaks for $\alpha=10^{\circ}$ and $20^{\circ}$ by $\tilde{t} \approx 4.5$, as is the case for other angles of attack. This is likely because the instantaneous Reynolds number needed to reach a low- $\alpha$ Hopf bifurcation in this accelerating flow is
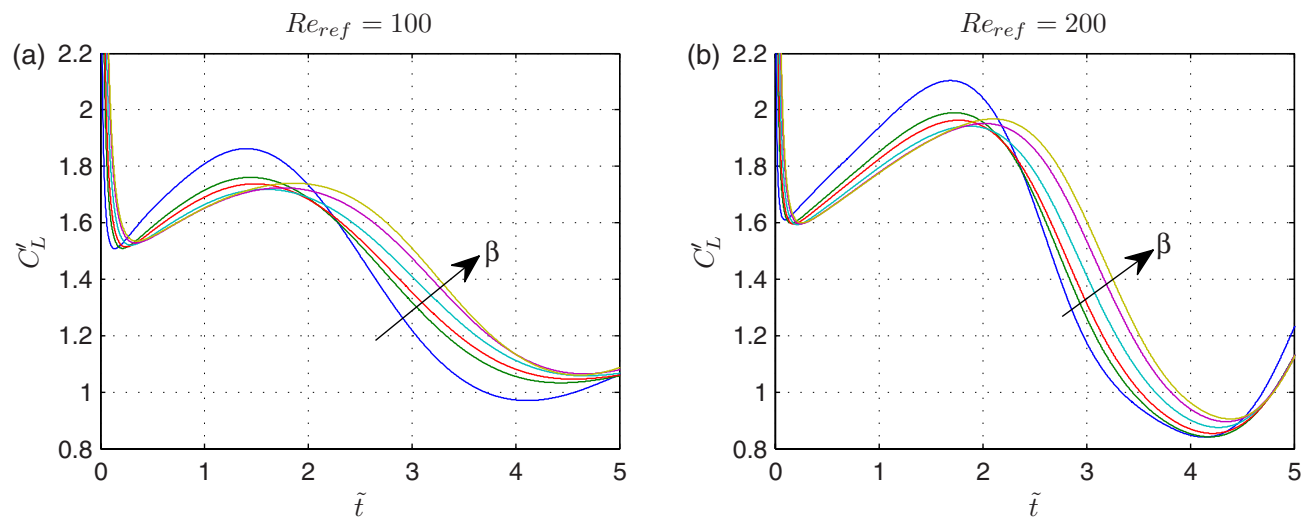

FIG. 5. (Color online) Lift augmentation curves with a focus on low powers, at $\alpha=30^{\circ}$. (a) This plot is similar to Fig. 4(a), but focuses on lower values of $\beta$. Shown are $\beta=0.0,0.2,0.3,0.5,0.8$, and 1.0 at $\operatorname{Re}_{\text {ref }}=100$. (b) At $\operatorname{Re}_{\text {ref }}=200$. 

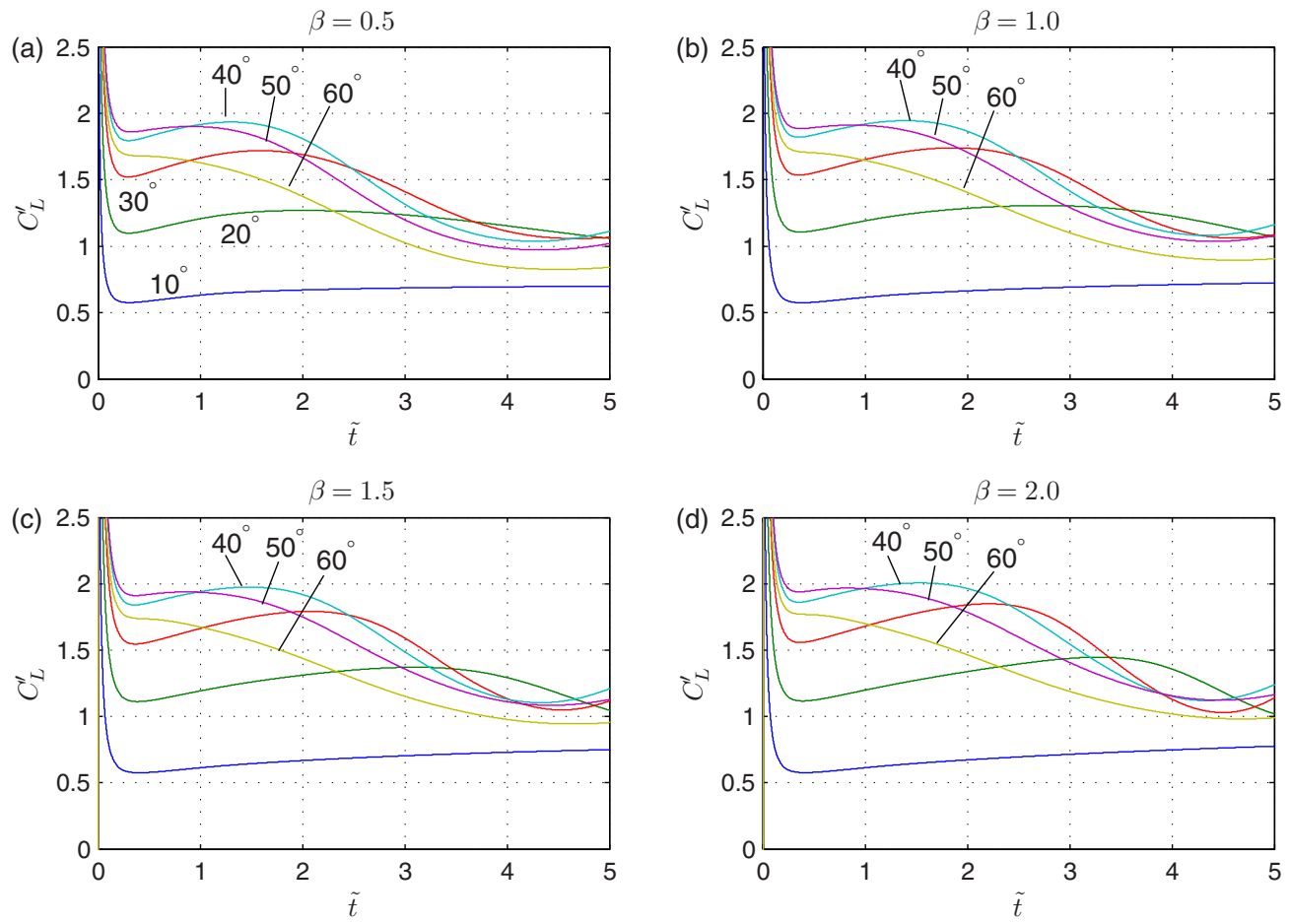

FIG. 6. (Color online) Effect of angle of attack on lift augmentation at $\operatorname{Re}_{\mathrm{ref}}=100$. (a) $\beta=0.5$, (b) $\beta=1.0$, (c) $\beta=1.5$, and (d) $\beta=2.0$.

higher than what is achieved at $\tilde{t} \approx 4.5$. Visualization of the computational results near $\tilde{t}=4.5$ shows a large but stable separation bubble over the flat plate. We suspect that at a higher $\mathrm{Re}_{\text {ref }}$, the time scale of the lift augmentation may match well with $\tilde{t}=4.5$ at $\alpha=20^{\circ}$ or below.

Second, the lift augmentation is maximized near $\alpha$ $=40^{\circ}$. When $\alpha$ approaches $60^{\circ}$, the high angle of attack causes the LEV's force augmentation to align more closely with the direction of drag. Although drag is known to play an important role in insect hovering, sometimes producing three quarters of the vertical force,${ }^{10}$ it will not be discussed here. Third, as in Figs. 4(a) and 5, the lift augmentation curves in Figs. 6(a)-6(d) are fairly similar across different values of $\beta$. The augmentation is slightly more pronounced at higher $\beta$, but in general, all angles of attack from $10^{\circ}$ to $60^{\circ}$ show only a weak sensitivity in lift augmentation to $\beta$. In particular, at $\alpha \geq 30^{\circ}$, the peaks consistently end around $\tilde{t}=4.5$, as in Fig. 4(d). Finally, at $\alpha \leq 30^{\circ}$, the lift coefficient at the onset of augmentation (i.e., the first minimum in $C_{L}$ or $C_{L}^{\prime}$ ) is very close to the lift predicted by Wagner's theory; this corresponds to $C_{L}^{\prime}=\pi \sin \alpha$. Wagner's theory becomes invalid for large $\alpha$.

The LEV formation process is visualized in Fig. 7 for $\beta=0.0,1.0$, and 5.0. Vorticity contours and streamlines outline the vortical and circulating structures from the onset of LEV formation (left column) to the point where the LEV sheds (middle column), and finally to the formation of the second LEV (right column). During the formation of the first LEV, positive vorticity is generated at the trailing edge and negative vorticity is generated at the leading edge. As theory predicts, the startup trailing-edge vortex is immediately shed into the flow. When the lift augmentation reaches its maximum, the LEV separates. It is noteworthy that at this separation point (middle column), $\operatorname{Re}=100$ at $\beta=0.0, \operatorname{Re}=193$ at $\beta=1.0$, and $\operatorname{Re}=967$ at $\beta=5.0$. Since the diffusion of vorticity is reduced at higher Reynolds numbers, the roll-up of the vortex sheet is clear for $\beta=5.0$ in the middle column. The same effect can be observed when the second LEV forms, at which point $\operatorname{Re}=100$ at $\beta=0.0, \operatorname{Re}=304$ at $\beta=1.0$, and $\operatorname{Re}=1612$ at $\beta=5.0$.

\section{Long-time vortex shedding}

After the first several LEVs have formed, a regular vortex shedding pattern establishes in the wake of the flat plate. This flow regime is referred to as a quasisteady state, because the bounds and period of the $C_{L}$ oscillation are not truly constant. Figure 8 (a) depicts the $C_{L}$ oscillation that occurs after the transient effects pass and a vortex shedding pattern establishes. Although the frequency $f$ of oscillation appears to be dependent on $\beta$, plotting a nondimensional frequency $\tilde{f} \equiv f(c \sin \alpha)^{2} / \nu$ against the instantaneous Reynolds number in Fig. 8(b) shows that the temporal nature of the vortex shedding pattern is determined largely by the instantaneous Reynolds number.

To calculate one value of $\tilde{f}$ per oscillation, we first separate out the Reynolds number, so that $\tilde{f}=f c \overline{\operatorname{Re}} \sin ^{2} \alpha / \bar{U}$. Both $\bar{U}$ and $\overline{\mathrm{Re}}$ are integrated averages through a period, that is,

$$
\begin{aligned}
\bar{U} & =\frac{1}{t_{2}-t_{1}} \int_{t_{1}}^{t_{2}} U d t \\
& =\frac{c\left(\tilde{t}_{2}-\tilde{t}_{1}\right)}{t_{2}-t_{1}},
\end{aligned}
$$



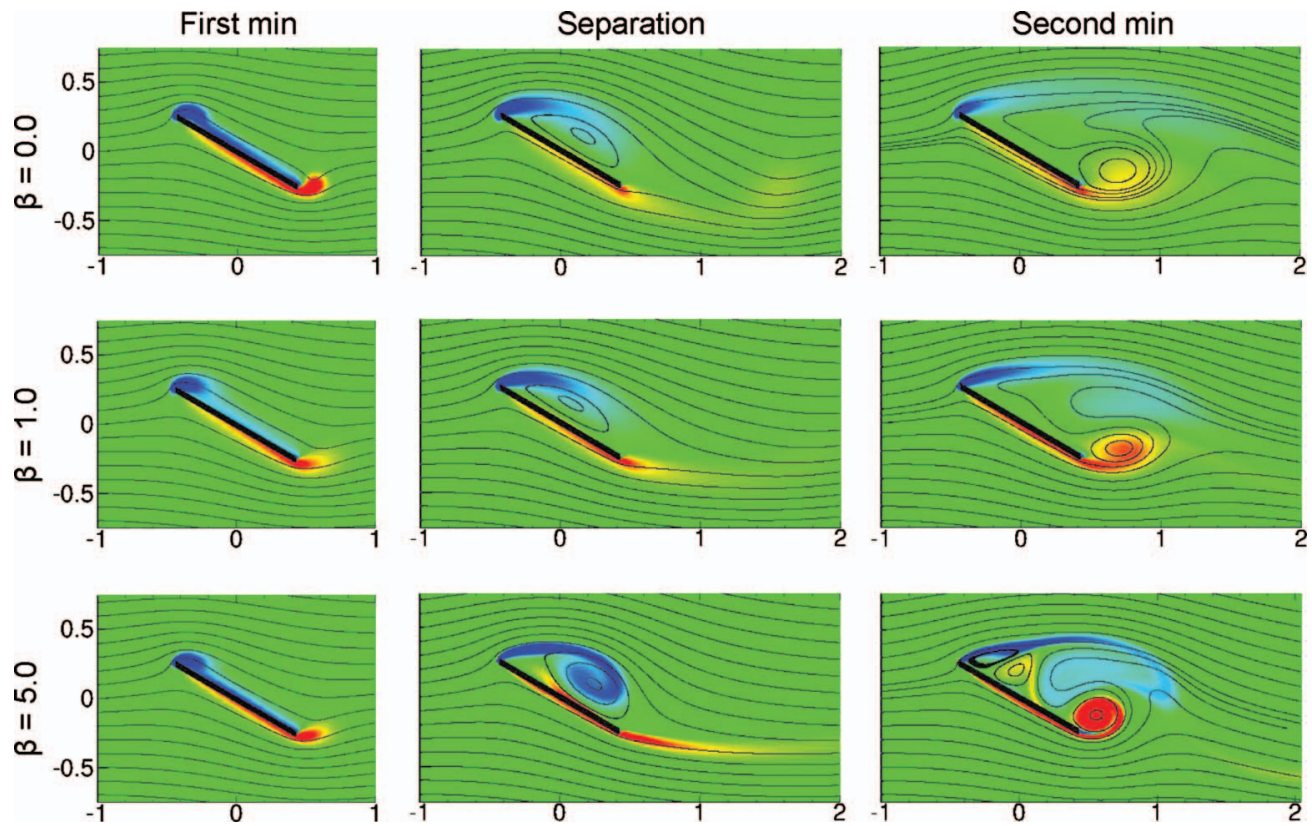

FIG. 7. (Color) Vorticity contours and streamlines at different stages of the initial LEV with $\operatorname{Re}_{\text {ref }}=100$ and $\alpha=30^{\circ}$. Left column: the first minimum in $C_{L}^{\prime}$, where the LEV begins to form; middle column: the first maximum in $C_{L}^{\prime}$, where the LEV begins to separate; right column: the second minimum in $C_{L}^{\prime}$, where the second LEV begins to form. The contour coloring ranges from positive vorticity in red to negative in blue. The shading is normalized by the instantaneous $\hat{U}$ across all nine snapshots.

$$
\begin{aligned}
\overline{\operatorname{Re}} & =\frac{\operatorname{Re}_{\mathrm{ref}}}{t_{2}-t_{1}} \int_{t_{1}}^{t_{2}} \frac{U(t)}{U_{\mathrm{ref}}} d t \\
& =\operatorname{Re}_{\mathrm{ref}} \frac{\tilde{t}_{2}-\tilde{t}_{1}}{\hat{t}_{2}-\hat{t}_{1}} .
\end{aligned}
$$

Similarly, we take $f$ to be an average over a period. Hence,

$$
f=\frac{1}{t_{2}-t_{1}}
$$

and thus

$$
\tilde{f}=\frac{\operatorname{Re}_{\mathrm{ref}} \sin ^{2} \alpha}{\hat{t}_{2}-\hat{t}_{1}} .
$$

The linear relation between $\tilde{f}$ and Re in Fig. 8(b) is similar to the results found by Roshko ${ }^{11}$ and Sreenivasan et al. ${ }^{12}$ for a steady flow over a cylinder. In the case of an accelerating plate at $\mathrm{Re}_{\text {ref }}=100$ and $\alpha=30^{\circ}$, the relation between frequency and Reynolds number is given by

$$
\tilde{f}=0.0895 \overline{\operatorname{Re}}-2.605 .
$$

It is remarkable that this relation holds for both steady and accelerating airfoils.

The independence of acceleration from long-time dynamics is also apparent when the lift coefficient is viewed as a function of the instantaneous Reynolds number $\operatorname{Re}=\operatorname{Re}_{\text {ref }} \hat{U}$ as opposed to $\widetilde{t}$. In Fig. 9, the collective data for $0.1 \leq \beta \leq 1.5$ show a well-defined oscillation envelope across all $\operatorname{Re}_{\text {ref }}$ and $\beta$. Furthermore, the envelope coincides nearly exactly with the bifurcation limits given by constant-velocity simulations at $\operatorname{Re}=100,200,400$, and 800, which are shown by large circles in the figure. Although accelerations $d \hat{U} / d \hat{t}$
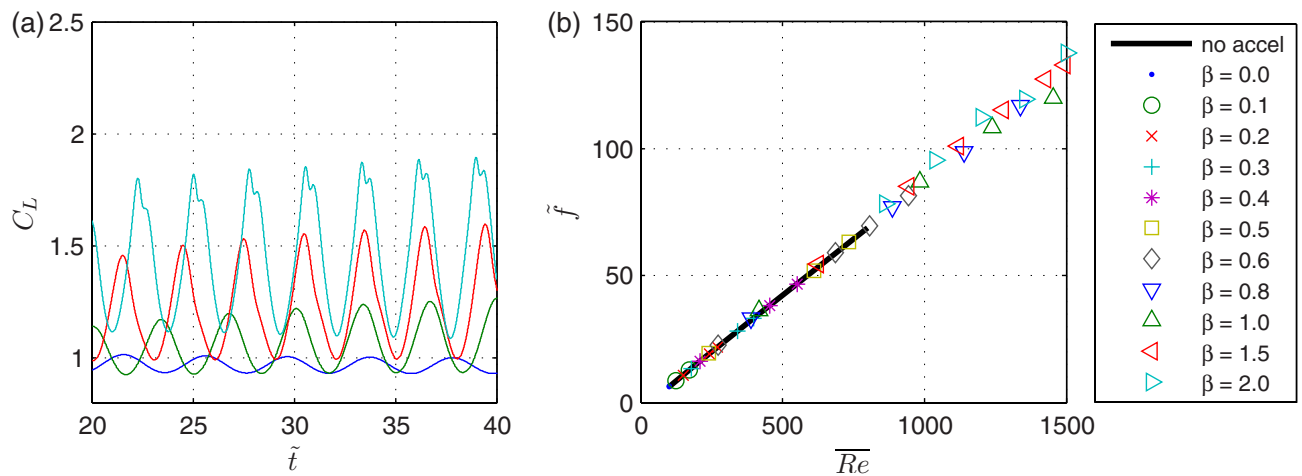

FIG. 8. (Color online) Analysis of quasisteady state conditions at $\mathrm{Re}_{\mathrm{ref}}=100$ and $\alpha=30^{\circ}$. (a) The lift coefficient $C_{L}$ during quasisteady state. This plot is a direct continuation of Fig. 2(d); Fig. 2(d) depicts $0<\tilde{t}<20$, and (a) here depicts $20<\tilde{t}<40$. From lowest amplitude to highest: $\beta=0.0,0.2,0.5$, and 1.5 . (b) The frequency of oscillation across different powers. 

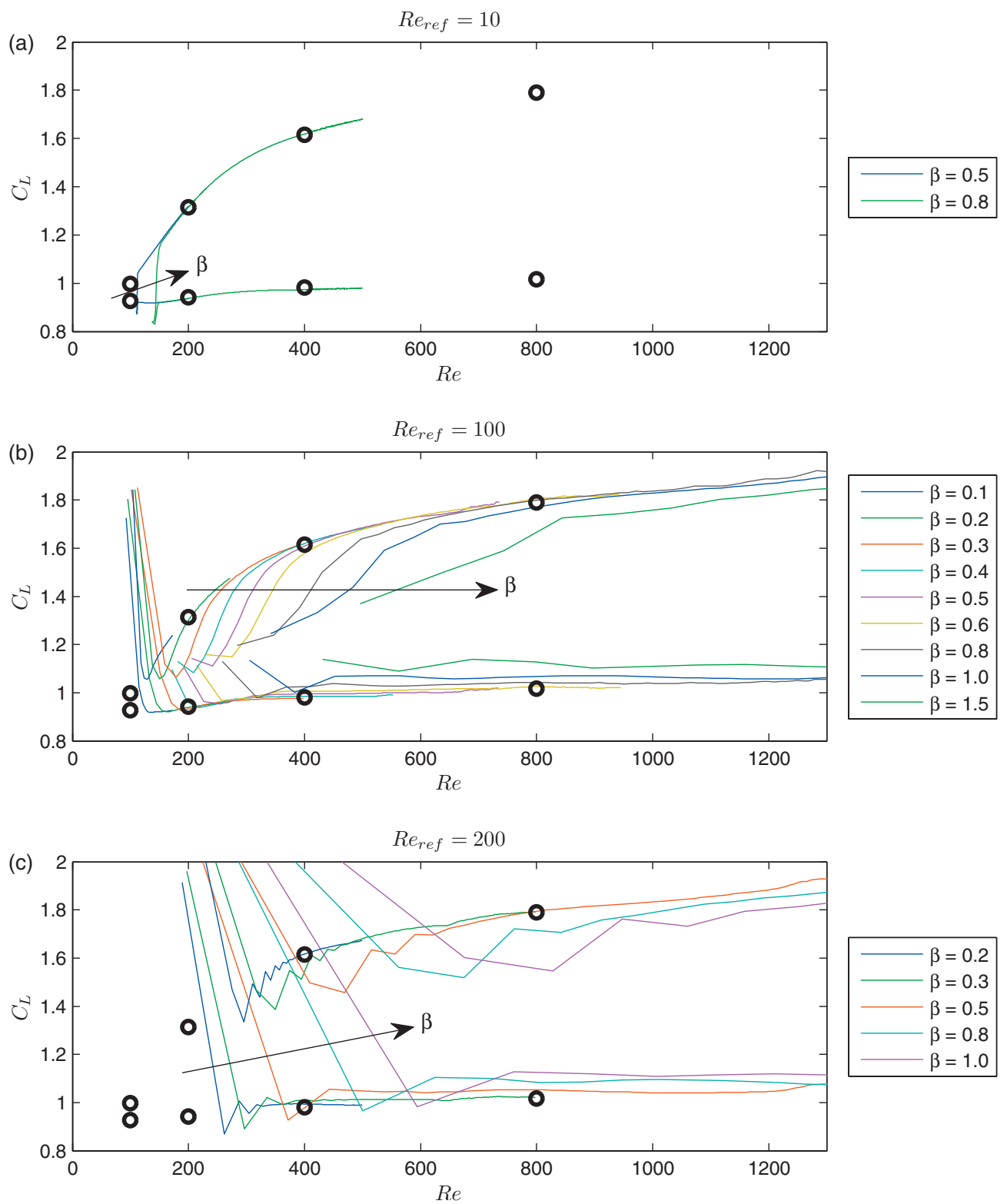

FIG. 9. (Color) Oscillation minima (bottom curves) and maxima (top curves) at various $\operatorname{Re}_{\text {ref }}$ and $\beta$, plotted as $C_{L}$ vs instantaneous Re. The circles indicate oscillation minima and maxima for impulsively accelerated runs, i.e., $\beta=0.0$. (a) $\operatorname{Re}_{\mathrm{ref}}=10$, (b) $\operatorname{Re}_{\mathrm{ref}}=100$, and (c) $\operatorname{Re}_{\mathrm{ref}}=200$.

in this figure range from less than 0.0008 to 1 and beyond, all lift coefficient curves across all reference Reynolds numbers collapse into the limits given by constant-velocity tests. This provides convincing evidence that it is the Reynolds number, not the acceleration, that determines the quasisteady state oscillation minimum and maximum. That is, whereas the quasisteady state model of transient airfoil acceleration fails at early times, such a model holds quite well once vortex shedding is well-established and transient effects have faded.

Both steady flows and accelerating flows exhibit an envelope in $C_{L}$ roughly shaped by a square root curve, particularly at low Re. This is predicted by the Hopf bifurcation. ${ }^{12}$ According to the bifurcation theory, the amplitude of oscillation is expected to be proportional to $\sqrt{R e}-\mathrm{Re}_{\text {crit }}$ near $R e=R_{\text {crit }}$, where $\operatorname{Re}_{\text {crit }}$ is the Reynolds number at which the wake becomes unsteady.

\section{FURTHER DISCUSSION}

Previous studies (e.g., Dickinson and Götz ${ }^{5}$ ) have suggested that the lift augmentation observed for an airfoil at moderate angle of attack and low Reynolds number is beneficial to insect flight. Dickinson and Götz observed the lift augmentation on an impulsively started airfoil and found the time scale of the peak lift to correspond well with the twoto-four chord-length half-stroke of fly wings. That is, flies can reap the full benefits of the lift augmentation through each half-stroke. The present study confirms that this benefit applies even when the airfoil is subjected to a range of accelerations, from an impulsive motion at $\beta=0$ to rapidly accelerating motion at $\beta=5$. That the lift augmentation time scale (or alternatively, displacement scale) $\tilde{t}$ is universal across power law accelerations is significant, because a true 

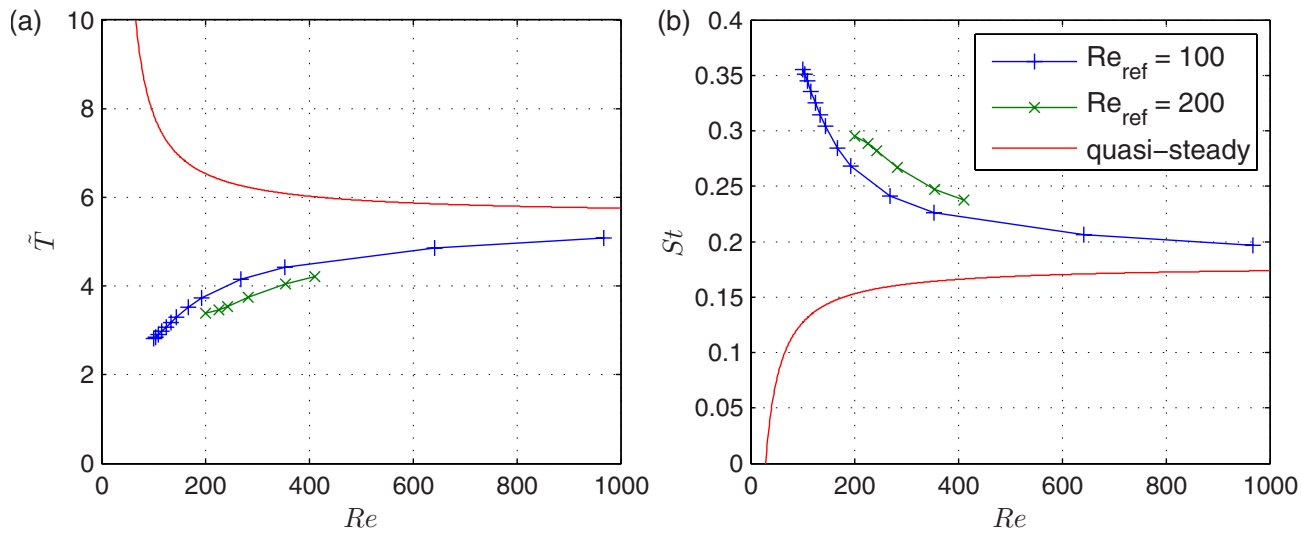

FIG. 10. (Color online) The Reynolds number dependence of the initial LEV formation time $\widetilde{T}$ at $\operatorname{Re}_{\text {ref }}=100$ and 200 . The formation time in the long-time response is shown for comparison. (a) Viewed in terms of $\widetilde{T}$. (b) Viewed in terms of Strouhal number St.

insect wing stroke is a complicated, constantly accelerating motion.

Parallels can be drawn between the present study and the concept of vortex formation time. ${ }^{13,14}$ This has been the subject of recent and ongoing research because a formation time of $\tilde{T} \approx 4$ has been observed in many vortex generating devices. ${ }^{13}$ For example, such a vortex formation time was first observed in piston-cylinder vortex ring generators, ${ }^{14}$ but the same $\widetilde{T} \approx 4$ was also observed in flow over a cylinder ${ }^{15}$ and over flapping wings. ${ }^{16}$

Under the current setup, the vortex formation time is defined as

$$
\widetilde{T} \equiv \frac{1}{l_{0}} \int_{0}^{t_{\max }} U(t) d t
$$

where $l_{0}=c \sin \alpha$ is the obstruction length viewed by the oncoming flow and $t_{\max }$ is the time at which the vortex separates from the airfoil. Incidentally, $\widetilde{T}$ is the exact inverse of the Strouhal number St, if we define

$$
\mathrm{St} \equiv \frac{f l_{0}}{\int_{0}^{t_{\max }} U(t) d t / t_{\max }},
$$

and $f=t_{\max }^{-1}$; compare Figs. 10(a) and 10(b). The vortex separation is assumed to occur when $C_{L}^{\prime}$ reaches a maximum. Computing the integral for power law velocities yields

$$
\widetilde{T}=\frac{\tilde{t}_{\max }}{\sin \alpha} .
$$

This is shown for $\mathrm{Re}_{\mathrm{ref}}=100$ and 200 in Fig. 10. At $\mathrm{Re}_{\mathrm{ref}}$ $=100$ and $\alpha=30^{\circ}$, we find that $\widetilde{T}$ ranges from 2.8 at $\beta=0.0$ to 5.1 at $\beta=5.0$. In the $0.0 \leq \beta \leq 1.0$ range, $2.8 \leq \widetilde{T} \leq 3.7$. Because $\sin \alpha=0.5$, these values can be obtained by multiplying the $\tilde{t}$ values of Fig. 4(d) by 2 . At $\operatorname{Re}_{\text {ref }}=200$ and 0.0 $\leq \beta \leq 1.0$, the vortex formation time ranges from 3.4 to 4.2 .

Extrapolating from Eq. (21), the vortex formation time in the quasisteady state (which, as previously discussed, is identical to that in the impulsively accelerated case) should asymptotically approach 5.6 as the Reynolds number becomes large. This, of course, ignores transition to turbulence and other high Reynolds number effects. At Re $>400$, Fig. 10(a) shows the asymptote on the quasisteady state $\widetilde{T}$ to support that vortex formation time. Once again using Eq. (21), as well as Fig. 8(b), however, we also find that $\widetilde{T}$ becomes much larger at low Reynolds numbers. In this Re range, the dependence of $\widetilde{T}$ on Re is very significant. Previous studies in the vortex formation time typically employed higher Reynolds numbers than in this study, ${ }^{14-16}$ so Reynolds number effects there may not be apparent.

The formation time of the initial LEV, like the vortex formation time in the quasisteady state, is heavily dependent on Re at low Re and less so at high Re. It is crucial to note, however, that the dependence on Re runs in the opposite direction as compared with the quasisteady state formation time. Whereas $\widetilde{T}$ increases in the quasisteady state for lower values of Re, the formation time of the first LEV decreases. This is a counterintuitive result that highlights the fundamental difference between the initial and subsequent large-time LEV formations.

\section{CONCLUSION}

We computed the 2D flow over a flat plate accelerated through ambient fluid at a speed $\hat{U}=\hat{t}^{\beta}$, focusing on $\mathrm{Re}_{\text {ref }}=100$ and an angle of attack of $\alpha=30^{\circ}$. Values of $\beta$ ranged from 0 to 5 . All cases exhibited an early transient lift coefficient governed by the added mass lift $C_{L} \approx \pi \sin (2 \alpha) \beta /(4(\beta+1) \widetilde{t})$. In addition, in all cases the viscous contribution to $C_{L}$ peaked immediately following the early transient $C_{L} \sim \widetilde{t}^{-1}$ relation. For a given Reynolds number and angle of attack, the peaks collapsed fairly well at $0.0 \leq \beta \leq 1.0$, but carried some noticeable dependence on $\beta$ for $\beta>1$.0. Nevertheless, for all values of $\beta$ in $0 \leq \beta \leq 5$ and for $\alpha \geq 30^{\circ}$, the lift augmentation associated with the LEV concluded at about 4.5 chord lengths. Given a typical halfstroke of two to four chord lengths for insect wings, this reinforced the theory that insects take advantage of the observed lift augmentation to aid their flight. ${ }^{5}$ At lower angles of attack, the lift augmentation was prolonged because higher Reynolds numbers were needed to induce shedding; at higher angles of attack, the augmentation contributed more to drag than to lift. 
After the airfoil completed the startup stage, a quasisteady pattern of vortex shedding established. The period of oscillation was characterized by a relation between the nondimensional frequency and the instantaneous Reynolds number, and this relation coincided with that of the constantvelocity case. In addition, the bounds of the force oscillation overlapped across all $\beta$ when the lift coefficient was viewed as a function of the instantaneous Reynolds number. These bounds were consistent with the bounds computed from constant-velocity runs at the same angle of attack. This led to the conclusion that after transient effects have passed, the nature of the vortex shedding and the consequent dynamics were determined primarily by the instantaneous velocity, and that acceleration produced no noticeable effect.

Finally, the notion of a vortex formation time was applied to the computational results. A universal formation time of $\widetilde{T} \approx 4$ had been previously observed in piston-cylinder setups, in flow over a cylinder, and in flow over flapping wings. Here, the formation time of the initial LEV increased with Re and the formation time in the quasisteady state decreased with $\mathrm{Re}$, both particularly in the low Reynolds number range. Even with the dependence on Re, however, $\widetilde{T}$ remained close to 4 for the initial LEV.

\section{ACKNOWLEDGMENTS}

We would like to thank Professor David Williams for his insightful suggestions. This work was supported in part by AFOSR Grant No. FA9550-05-1-0369 (T.C. and K.T.) and Grant No. FA9550-09-1-0189 (T.C.).
${ }^{1}$ J. M. Birch, W. B. Dickson, and M. H. Dickinson, "Force production and flow structure of the leading edge vortex on flapping wings at high and low Reynolds numbers," J. Exp. Biol. 207, 1063 (2004).

${ }^{2}$ S. P. Sane, "The aerodynamics of insect flight," J. Exp. Biol. 206, 4191 (2003).

${ }^{3}$ J. J. Videler, E. J. Stamhuis, and G. D. E. Povel, "Leading-edge vortex lifts swifts," Science 306, 1960 (2004).

${ }^{4}$ K. Taira and T. Colonius, "Three-dimensional flows around low-aspectratio flat-plate wings at low Reynolds numbers," J. Fluid Mech. 623, 187 (2009).

${ }^{5}$ M. H. Dickinson and K. G. Götz, "Unsteady aerodynamic performance of model wings at low Reynolds numbers," J. Exp. Biol. 174, 45 (1993).

${ }^{6}$ D. I. Pullin and Z. J. Wang, "Unsteady forces on an accelerating plate and application to hovering insect flight," J. Fluid Mech. 509, 1 (2004).

${ }^{7}$ H. Wagner, "Über die Entstehung des dynamischen Auftriebes von Tragflüeln," Z. Angew. Math. Mech. 5, 17 (1925).

${ }^{8} \mathrm{~K}$. Taira and T. Colonius, "The immersed boundary method: A projection approach,” J. Comput. Phys. 225, 2118 (2007)

${ }^{9}$ T. Colonius and K. Taira, "A fast immersed boundary method using a nullspace approach and multi-domain far-field boundary conditions," Comput. Methods Appl. Mech. Eng. 197, 2131 (2008).

${ }^{10}$ Z. J. Wang, "The role of drag in insect hovering," J. Exp. Biol. 207, 4147 (2004).

${ }^{11}$ A. Roshko, "On the development of turbulent wakes from vortex streets," NACA Report No. 1191, 1954.

${ }^{12}$ K. R. Sreenivasan, P. J. Strykowski, and D. J. Olinger, "Hopf bifurcation, Landau equation, and vortex shedding behind circular cylinders," paper presented at the Forum on Unsteady Flow Separation at the 1987 ASME Applied Mechanics, Bioengineering, and Fluids Engineering Conference, Cincinnati, OH, 14-17 June 1987.

${ }^{13}$ J. O. Dabiri, "Optimal vortex formation as a unifying principle in biological propulsion,” Annu. Rev. Fluid Mech. 41, 17 (2009).

${ }^{14} \mathrm{M}$. Gharib, E. Rambod, and K. Shariff, "A universal time scale for vortex ring formation,” J. Fluid Mech. 360, 121 (1998).

${ }^{15}$ D. Jeon and M. Gharib, "On the relationship between the vortex formation process and cylinder wake vortex patterns," J. Fluid Mech. 519, 161 (2004).

${ }^{16}$ M. Milano and M. Gharib, "Uncovering the physics of flapping flat plates with artificial evolution,” J. Fluid Mech. 534, 403 (2005). 